\title{
Iatrogenic scleroderma renal crisis: A case report and mini literature review for renal crisis
}

\author{
Gülșah Boz ${ }^{1}$, Samet Karahan*2, Nurdan Yüksek ${ }^{3}$ \\ ${ }^{1}$ Kayseri City Education and Research Hospital, Department of Internal Medicine, Division of Nephrology, Kayseri, Turkey \\ ${ }^{2}$ Kayseri City Education and Research Hospital, Department of Internal Medicine, Division of Rheumatology, Kayseri, Turkey \\ ${ }^{3}$ Kayseri City Education and Research Hospital, Department of Internal Medicine, Kayseri, Turkey
}

Received: June 2, 2021

DOI: $10.5430 / \mathrm{dcc} . \mathrm{v} 8 \mathrm{n} 3 \mathrm{p} 4$
Accepted: August 4, 2021

URL: https://doi.org/10.5430/dcc.v8n3p4
Online Published: September 26, 2021

\begin{abstract}
Background: Systemic sclerosis is a connective tissue disease characterized by fibrosis of the skin and organs, marked changes in microvascular structure, cellular and humoral immune disorders. Renal involvement is more frequent and mainly characterized by moderate proteinuria, elevated serum creatinine levels, and hypertension. The most common kidney involvement in SSc is scleroderma renal crisis (SRC) that is fatal without prompt intervention.

Case report: A 52-year-old Caucasian male with known diffuse cutaneous systemic sclerosis was hospitalized with communityacquired pneumonia. On the fifth day after appropriate antibiotic therapy and $60 \mathrm{mg} /$ day methylprednisolone, decreased urine output, arterial hypertension, decreased renal function and pulmonary edema developed. The patient was diagnosed with a scleroderma renal crisis. Emergency hemodialysis was applied to the patient, and captopril $6 \times 25 \mathrm{mg} /$ day and nifedipine $2 * 60$ $\mathrm{mg} /$ day treatment were given. He received a routine hemodialysis program for about three months. The hemodialysis program was terminated when the patient's urine quality and quantity increased.

Conclusions: SRC, characterized by malignant hypertension, azotemia, microangiopathic hemolytic anemia, and kidney failure, is one of the most important complications of systemic sclerosis with a poor prognosis without prompt intervention. Steroid use is one of the important risk factors that precipitate SRC development. With angiotensin-converting enzyme inhibitors, survival increased after SRC, the need for dialysis decreased, and usually allowed the discontinuation of dialysis treatment within about 6-18 months. Suspicion of SRC in the presence of the above-mentioned findings in patients with a diagnosis or suspected systemic sclerosis can be considered the most important treatment step.
\end{abstract}

Key Words: Scleroderma, Systemic sclerosis, Scleroderma renal crisis, Systemic sclerosis-associated thrombotic microangiopathy

\section{INTRODUCTION}

Systemic sclerosis ( $\mathrm{SSc}$ ) is a rare, clinically heterogeneous, chronic inflammatory disease characterized by fibrosis of the skin and internal organs. Although the pathogenesis of the disease is not clearly known, much evidence of vasculopathy and immune activation is presented. ${ }^{[1]}$ Scleroderma renal involvement, as manifested by kidney failure, was described first in 1863. Over the next 80 years, numerous case reports linked scleroderma with renal disease and increased mortality. However, by 1952 Moore and Sheehan had described the kidney lesion as the leading cause of death in scleroderma. ${ }^{[2]}$ The pathology is well described, and it is known that the

\footnotetext{
*Correspondence: Samet Karahan; Email: doktorsamet@yahoo.com; Address: Kayseri City Education and Research Hospital, Department of Internal Medicine, Division of Nephrology, Kayseri, Turkey.
} 
renin-angiotensin system plays an important role in this complication; the reasons why there is a predilection for patients with diffuse scleroderma and the triggers of renal damage are still unknown. However, with a well-defined pathology, it has been understood that it is important to diagnose scleroderma renal crisis as early as possible, thus preventing kidney damage by rapid therapeutic intervention. In this case report, we wanted to emphasize the scleroderma renal crisis (SRC) phenomenon, where early therapeutic intervention is life-saving.

\section{CASE REPORT}

A 52-year-old Caucasian male patient, known to have diffuse cutaneous systemic sclerosis, applied to the emergency department with fever, chills, sweating, and increased sputum, and he had a fever of $39^{\circ} \mathrm{C}$ and coarse crackles in the right middle zone. Infiltrates were also detected in the aforementioned lung area of the chest X-ray of the patient, who was learned to have chronic kidney disease and whose basal creatinine level was $1.5 \mathrm{mg} / \mathrm{dl}$ was seen from the old medical records. The patient was hospitalized with the diagnosis of community-acquired pneumonia, and ampicillin-sulbactam + clarithromycin and metilpredinozolone (60 mg/day) was started for pneumonia by the pulmonology and tuberculosis clinic. Although not specified as a standard treatment in the national treatment guidelines for community-acquired pneumonia, methylprednisolone is included in the treatment by the treating physician. The patient, who was working in the industry sector, had a diagnosis of systemic sclerosis for about nine years, did not use medication despite being recommended, and the patient did not tell the treating doctor his diagnosis clearly. On the $5^{t h}$ day of the treatment, a decrease in urine output, malignant arterial hypertension, decreased kidney function was observed, and the patient was consulted to nephrology. On physical examination, the patient looked agitated, hands had a sclerodactyly, but no digital ulcers (see Figure 1), and the face had a fish mouth appearance and a small pinched nose. His blood pressure was 220/120 $\mathrm{mmHg}$, the cardiac rate was $106 / \mathrm{min}, \mathrm{S} 1$ and S2 were hard, there was no additional murmur, exhalation time was long, and he had bibasilar crepitant rales up to mid-upper zones. Abdominal auscultation was non-remarkable, and hepatosplenomegaly was not detected. Complete blood count parameters were as follows, leukocyte $8,200 / \mathrm{mm}^{3}$, hemoglobin: $11.7 \mathrm{~g} / \mathrm{dl}$, hematocrit: $35 \%$ thrombocyte: $82.000 / \mathrm{mm}^{3}$, MCV: $90 \mathrm{fL} . \mathrm{mmol} / \mathrm{L}$ Nutritional parameters of the patient with thrombocytopenia and anemia are compatible with chronic disease anemia. In addition, there were no schistosomes and normochromic normocyte anemia in the peripheral smear. In urine analysis, hematuria and urinary casts were not found, and the protein

Published by Sciedu Press ratio was calculated as $620 \mathrm{mg} /$ day in spot urine. In terms of malignant hypertension and Sjögren syndrome, detailed eye examination was done and bilateral papillary edema accompanied by hemorrhage and exudate and bilateral grade 4 hypertensive retinopathy were observed in the eye examination and also Schirmer test was normal for both eyes. In the urinary system ultrasound, the right kidney was $85 \mathrm{~mm}$, the left $100 \mathrm{~mm}$, and there was a grade 2 echo increase in both kidney parenchyma, and the bladder and other collecting systems were normal. Bilateral renal artery and veins flow patterns were normal, and no sign of stenosis was observed. The patient was referred to the nephrology clinic with the diagnosis of scleroderma renal crisis and pulmonary edema after steroid therapy. Captopril $6 \times 25 \mathrm{mg} /$ day and nifedipine $2 \times 60 \mathrm{mg} /$ day immediately started. Although the patient had pulmonary edema, diuretics and intravenous nitroprusside were not given to the patient to prevent hypovolemia and rapid blood pressure decrease. Also, hemodialysis was applied to the patient because the patient's urine output was very low. The patient, whose breathing returned to normal on the second day and whose blood pressure did not return to normal levels, but was reduced, also scanned for interstitial lung disease. In respiratory function tests, both forced expiratory volume in 1 second (FEV1) and vital functional capacity (FVC) were decreased, and it was characterized as a restrictive ventilation defect. High-resolution lung tomography showed increased reticular density in the bilateral lung posterodorsal, atelectasis, and ground-glass appearance in the bilateral lung lower lobe. Significant dilatation was observed in the middle part of the esophagus and the pleural effusion was $35 \mathrm{~mm}$ on the right and $15 \mathrm{~mm}$ on the left. In the patient whose carbon monoxide diffusion capacity (DLCO) was found to be $48 \%$, additional suspicion was raised about the presence of pulmonary hypertension. On the patient's transthoracic echocardiography, ejection fraction was $60 \%$, minimal mitral insufficiency and minimal pericardial effusion were present, and mean pulmonary arterial pressure was $30 \mathrm{mmHg}$.

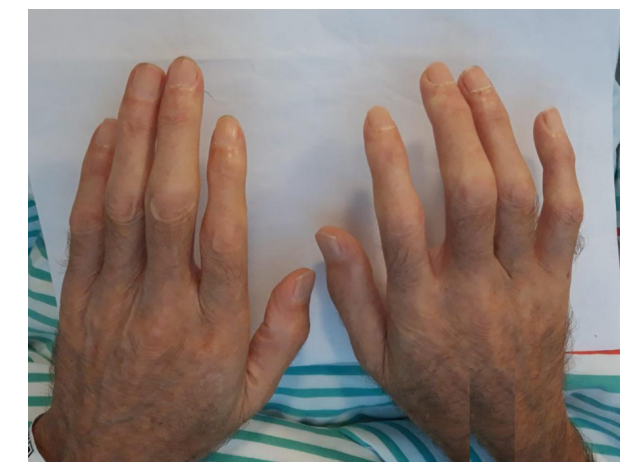

Figure 1. Sclerodactylic appearance of the patient's hand 
The anti-nuclear antibody test was positive in the granular pattern at 1/1,000 titration, but no autoantibody was found positive in the extractable nuclear antibody test performed by the immunoblot method. There were widespread active bleeding areas in capillaroscopic evaluation, avascular areas, giant capillaries, and tortiotic bizarre-shaped capillaries (see Figure 2). In the first week of hemodialysis treatment, the patient showed a normotensive course in the following days. However, due to poor quality urine output, elevated creati- nine levels, electrolyte imbalance, permanent hemodialysis catheterization, and routine dialysis program were approved. The patient, who did not need hemodialysis three months later, was removed from the hemodialysis catheter and was followed up in the outpatient clinic. Rheumatology and hematology outpatient clinics follow the patient with hydroxychloroquine sulfate $2 \times 200 \mathrm{mg} /$ day, acetylsalicylic acid $100 \mathrm{mg}$ day, nifedipine extended-release tablets $60 \mathrm{mg} / \mathrm{day}$, ramipril $5 \mathrm{mg} /$ day, and methotrexate $15 \mathrm{mg} /$ week.
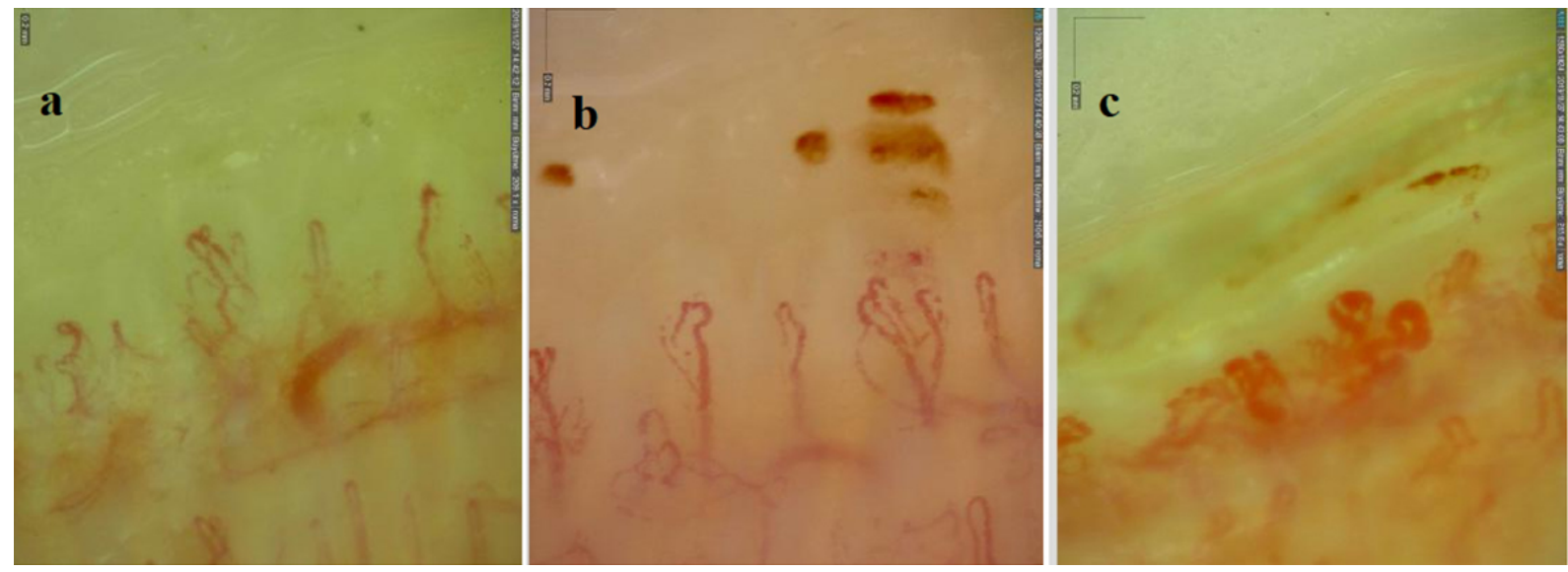

Figure 2. Nail Fold Video capillaroscopic findings of the patient's hand a) Bizarre-shaped, tortoise, and enlarged veins, b) Branching vessel and inactive old bleeding area at the tip, c) Giant capillaries

\section{DISCUSSION}

Scleroderma is a rare chronic inflammatory disease characterized by heterogeneous fibrosis of the skin and internal organs. Although the pathogenesis of the disease is not known clearly, the role of vasculopathy and immune activation has been determined. The disease is most common in the 30-50 years old, and the female/male ratio is $8 / 1 .^{[3]}$ Scleroderma is divided into two main groups as localized and systemic scleroderma ( $\mathrm{SSc}$ ). SSc is divided into three subgroups: diffuse cutaneous SSc (dcSSc), limited cutaneous SSs (lcSSc), and SSc sine-scleroderma. One of the major complications of SSc is SRC caused by the activation of the renin-angiotensin system due to damage to the renal microvascular system. SRC is characterized by sudden onset arterial hypertension and acute oliguric renal failure during SSc. Microangiopathic hemolytic anemia secondary to malignant hypertension, heart failure, pulmonary edema, hypertensive retinopathy, and encephalopathy can be seen in the course of the disease. ${ }^{[4]}$ Based on the regular follow-up data of the German Systemic Scleroderma Network (DNSS) in 2019 , SRC was developed in 70 of 2,873 patients $(2.4 \%)$ as a result of the analysis of data from 2,873 patients of Pia Monzaideh et al. with an average follow-up time of 3.8 years (2.4\%). DcSSc was diagnosed in $57.1 \%$ of these patients, lcSSc in $31.4 \%$, and overlap syndromes in $11.4 \% .^{[5]}$ Pre- vious studies noted that patients with dcSSc had a higher risk of up to $25 \%$ with SRC. ${ }^{[6]}$ SRC was observed in only $1 \%$ of patients with lcSSc. ${ }^{[6]}$ Some predictive features have been identified for SRC in patients with dcSSc. These predictors include being in the first year of illness, recent onset or disappearance of Raynaud's phenomenon, sudden onset of symptoms such as fatigue, weight loss and polyarthritis, swelling of the hands and lower legs, carpal tunnel syndrome, significant thickening of the arms, presence of antitopoisomerase I (anti-scl70 is another name of this antibody) and RNA polymerase III antibodies, glucocorticoid use more than $40 \mathrm{mg} /$ day prednisolone or equivalent and male gender. Our patient had methylprednisolone use of $60 \mathrm{mg} /$ day and male gender, which are one of the relevant predictors.

Antibodies can also predict SRC development, as mentioned above. Antitopoisomerase antibody is used as a marker for $\mathrm{dcSSc}$ as well as renal crisis, but its association with increased SRC has not been found. On the other hand, the anti-centromere antibody has been identified in a very small number of patients. ${ }^{[6]}$ While anti-topoisomerase antibodypositive patients developed SRC of about 10\%, anti-RNA polymerase III-positive patients had SRC of around $24 \%$ to $33 \%$. The independent risk factor with the highest probability of SRC for autoantibodies is positive anti-RNA polymerase 
III antibodies. ${ }^{[5]}$ In our case, ANA was positive in 1/1000 granular pattern; anti-topoisomerase I, anti-centromere, and anti RNA polymerase III were negative, and also regarding antiphospholipid antibodies, anti-cardiolipin IgG and IgM, anti-beta 2 glycoprotein $\operatorname{IgA}, \operatorname{IgM}$, and $\operatorname{IgG}$ were negative as well as lupus anticoagulant. When evaluated clinically, features related to kidney function were not helpful in predicting the renal crisis. Generally, normal blood pressure has been documented in patients 24 hours prior to the onset of SRC. Extremely high blood pressure develops suddenly. ${ }^{[5]}$ Although patients with scleroderma develop hypertension during the course of the disease, it is not more common than this general population and does not predict future SRC. ${ }^{[6]}$ Our patient had no known hypertension before SRC.

In recent years, $\mathrm{SSc}$ and scleroderma-like disease have been associated with exposure to other substances in industrial areas. Silica powders, vinyl chloride, L-tryptophan, breast silicone implants, and organic solvents are environmental factors associated with scleroderma. ${ }^{[7]}$ Our patient also works in the industrial sector, and it is predicted that occupational exposure may be involved.

In experimental studies, it was observed that glucocorticoids cause damage to the vascular endothelium and cause nephrosclerosis. In a retrospective multicenter study in 2012, 91 patients with SRC and 427 patients with non-SRC-SSc were compared. Patients in the SRC group received statistically significantly more prednisone 29.3 (28.4) vs. 3.6 (9.9) $\mathrm{mg}$ compared to non-SRCs. ${ }^{[8]}$ In one cohort of $50 \mathrm{SSc}$ patients with SRC, 30 (60\%) had been exposed to corticosteroids before SRC started. ${ }^{[9]}$ In another systematic review conducted by Trang et al., they examined data from 26 studies of $500 \mathrm{SSc}$ patients who received new corticosteroid therapy. Only ten of them are definite cases of SRC, and all of these ten patients received medium-to-high dose corticosteroid therapy. ${ }^{[10]}$ It was reported that glucocorticoids suppress prostacyclin production and increase angiotensinconverting enzyme (ACE) activity, causing renovascular damage. ${ }^{[11]}$ It is also important to differentiate between SRC and thrombotic microangiopathy (TMA) because TMA is observed in approximately $50 \%$ of SRC patients. In this context, Yamashita et al. have recently proposed defining SSc-associated TMA as a pathophysiological and clinical entity truly distinct from SRC. ${ }^{[12]}$ The authors also presented the following as a suggestion in order to make the distinction; they thought that the first complaint of SSc-TMA patients was thrombocytopenia, and increased blood pressure and worsening of renal function appeared later. On the other hand, SRC appears to be characterized by markedly elevated blood pressure and worsening of renal function, followed by mild thrombocytopenia. As explained above, although the effects of steroids on SRC are controversial, the sudden onset of SRC in this patient without pre-existing hypertension suggests that it may be a steroid-induced and iatrogenic SRC.

The SRC almost always had a fatal outcome before ACE inhibitors (and/or angiotensin receptor blockers - ARBs) were used in therapy. Less than $10 \%$ of patients who developed $\mathrm{SRC}$ at that time survived for more than three months. Few survivors were reported in the late 1970s with aggressive control of blood pressure and dialysis use. ${ }^{[13]}$ ACE inhibitors act as competitive inhibitors in the conversion of angiotensin I to angiotensin II. Inhibition of angiotensin II production rapidly lowers blood pressure in patients with scleroderma. Although angiotensin I and renin continue to accumulate, they are not biologically active and do not affect blood pressure. These drugs also provide proteolysis of bradykinins that cause hypotension with a strong vasodilator effect. ${ }^{[14]}$ While the first-year survival was achieved in less than $10 \%$ of patients before the use of ACE inhibitors, 5-year survival after the use of these drugs increased to $65 \% .{ }^{[4]}$ In some studies, initiating ACE inhibitor therapy has been associated with a poor outcome when serum creatinine level is 3 and above. ${ }^{[15]}$ Patients whose blood pressure cannot be controlled within three days are less likely to get good results. Male gender, advanced age, and congestive heart failure are other additional conditions associated with a poor outcome. ${ }^{[4]}$ Management is difficult in these patients, even by dialysis, in the presence of uncontrolled resistant hypertension, hyperreninemia, and congestive heart failure. In ancient times, bilateral nephrectomy was performed to block the renin-angiotensin-aldosterone pathway in SRC and to eliminate hyperreninemia. In this way, it has been noted that heart failure and dialysis management are more successful. ${ }^{[16]}$

Restoration of renal function after SRC may take up to three years (most commonly between 12-18 months). According to 2019 data on patients treated with ACE inhibitors from centers specialized in SSc care on all continents, ACE inhibitors can improve prognosis, but it is still unknown whether it prevents SRC. Dialysis is required in patients with kidney function loss. Despite the effectiveness of ACE inhibitors, SRC patients require approximately 20\%-50\% dialysis. ${ }^{[10]}$ Half of the patients may be free from dialysis during follow-up. Mortality and complication rates are higher in dialysis-dependent scleroderma.

\section{Conclusion}

SRC, characterized by malignant hypertension, hyperreninemia, azotemia, microangiopathic hemolytic anemia, and kidney failure, is one of the most important complications of 
systemic sclerosis with a poor prognosis without prompt intervention. We do not see scleroderma renal crisis much now because we treat hypertension in scleroderma early, immunosuppress early, especially with mycophenolate, and we avoid the use of any substantial corticosteroids in scleroderma. To avoid similar iatrogenic complications, clinicians should be educated/informed about avoiding the use of any substantial corticosteroids in scleroderma. Despite ACE inhibitor / ARBs and hemodialysis, it is one of the most important com- plications of SSc with a poor prognosis. Steroid use is one of the important risk factors that precipitate SRC development. With ACE inhibitors, survival increased after SRC, the need for dialysis decreased, and usually allowed the discontinuation of dialysis treatment within about 6-18 months. As in our case, we wanted to emphasize that early/rapid diagnosis and appropriate treatment can contribute to survival in SRC.

\section{CONFLiCTS OF INTEREST Disclosure}

The authors declare they have no conflicts of interest.

\section{REFERENCES}

[1] Rongioletti F, Ferreli C, Atzori L, et al. Scleroderma with an update about clinico-pathological correlation. G Ital di dermatologia e Venereol organo Uff Soc Ital di dermatologia e Sifilogr. 2018 Apr; 153(2): 208-15. https://doi.org/10.23736/S0392-0488.18 $.05922-9$

[2] Moore H, Sheehan H. The kidney of scleroderma. Lancet (London, England). 1952 Jan; 1(6698): 68-70. https://doi.org/10.101 6/s0140-6736(52) 91798-4

[3] Ingegnoli F, Ughi N, Mihai C. Update on the epidemiology, risk factors, and disease outcomes of systemic sclerosis. Best Pract Res Clin Rheumatol [Internet]. 2018; 32(2): 223-40. https://doi.or g/10.1016/j. berh. 2018.08.005

[4] Steen VD. Scleroderma renal crisis. Rheum Dis Clin North Am. 2003 May; 29(2): 315-33. https://doi.org/10.1016/s0889-8 $57 \times(03) 00016-4$

[5] Moinzadeh P, Kuhr K, Siegert E, et al. Scleroderma Renal Crisis: Risk Factors for an Increasingly Rare Organ Complication. J Rheumatol. 2020 Feb; 47(2): 241-8. https: //doi .org/10 . 3899/jrheum .180582

[6] Xu D, Zhu L, Cai R, et al. A multi-predictor model to predict risk of scleroderma renal crisis in systemic sclerosis: a multicentre, retrospective, cohort study. Clin Exp Rheumatol. 2021 May.

[7] Ouchene L, Muntyanu A, Lavoué J, et al. Toward Understanding of Environmental Risk Factors in Systemic Sclerosis [Formula: see text]. J Cutan Med Surg. 2021; 25(2): 188-204. https://doi.or $\mathrm{g} / 10.1177 / 1203475420957950$

[8] Guillevin L, Bérezné A, Seror R, et al. Scleroderma renal crisis: a retrospective multicentre study on 91 patients and 427 controls. Rheumatology (Oxford). 2012 Mar; 51(3): 460-7. https: //doi.org/10.1093/rheumatology/ker271
[9] Teixeira L, Mouthon L, Mahr A, et al. Mortality and risk factors of scleroderma renal crisis: a French retrospective study of 50 patients. Ann Rheum Dis. 2008 Jan; 67(1): 110-6. https: //doi.org/10.1136/ard.2006.066985

[10] Trang G, Steele R, Baron M, et al. Corticosteroids and the risk of scleroderma renal crisis: a systematic review. Rheumatol Int. 2012 Mar; 32(3): 645-53. https://doi.org/10.1007/s00296-010 $-1697-6$

[11] Zanatta E, Codullo V, Allanore Y. Scleroderma renal crisis: Case reports and update on critical issues. Eur J Rheumatol. 2020 Nov. https://doi.org/10.5152/eurjrheum.2020.20048

[12] Yamashita H, Kamei R, Kaneko H. Classifications of scleroderma renal crisis and reconsideration of its pathophysiology. Rheumatology (Oxford). 2019 Dec; 58(12): 2099-106. https://doi.org/10.1 093/rheumatology/kez435

[13] Wasner C, Cooke CR, Fries JF. Successful medical treatment of scleroderma renal crisis. N Engl J Med. 1978 Oct; 299(16): 873-5. https://doi.org/10.1056/NEJM197810192991607

[14] Jeong HS, Lim HS, Park HJ, et al. Clinical outcomes between calcium channel blockers and angiotensin receptor blockers in hypertensive patients without established cardiovascular diseases during a 3-year follow-up. Sci Rep. 2021 Jan; 11(1): 1783. https: //doi.org/10.1038/s41598-021-81373-7

[15] Lynch BM, Stern EP, Ong V, et al. UK Scleroderma Study Group (UKSSG) guidelines on the diagnosis and management of scleroderma renal crisis. Clin Exp Rheumatol. 2016; 34 Suppl 100(5): 106-9.

[16] Traub YM, Shapiro AP, Rodnan GP, et al. Hypertension and renal failure (scleroderma renal crisis) in progressive systemic sclerosis. Review of a 25-year experience with 68 cases. Medicine (Baltimore). 1983 Nov; 62(6): 335-52. https://doi.org/10.1097/000057 92-198311000-00001 